\title{
MACHINE BORNE INVENTIONS \& OWNERSHIP: A PATENTING PERSPECTIVE
}

\author{
Purwa Rathi \\ Senior Legal Counsel \\ Email: purwa.iplogy@gmail.com
}

\begin{abstract}
Artificial Intelligence (AI), in recent times, has been a hotly debated topic of interest worldwide. Various stakeholders, including the technologists, researchers, lawmakers, regulatory authorities, administration or the public in general are anxiously awaiting solutions to multifaceted challenges grappling AI industry today. Importantly, notions pertaining to legalities underpinning the evolution, emergence and execution of AI enabled technologies have gained widespread relevance. Amongst them, proprietary issues of inventorship, ownership, product liability etc. remain a key question. This primarily holds true for researchers interested in conceptualizing a new piece of intellectual work, establishing its ownership and eventually commercializing the same. Intellectual Property (IP) laws have been discretely administering the patent rules for 'natural persons'. However, determination of true proprietorship over technologies exclusively borne of a 'non-natural person' is setting a strong undercurrent within patent community today.

Many questions pertaining to proprietary interest in AI technologies have been baffling. Is the creator of AI machine also responsible for future uncertainties? How a machine is made accountable? Is it possible to confer a distinct identity to machine borne work? What may be the implications on existing patent regime? Will the unrealistic assessment of machine work by humans question the standards of patentability? If strict liabilities or penalties imposed on original creator of such machine stifle AI innovations in any way? The present study summarizes the assessment of factors influencing AI patent regime, potential challenges encountered by governing authorities during examination, and probable solutions for its reparation.
\end{abstract}

Key words: Machine Borne, Invention, Patenting, Artificial Intelligence, AI

Cite this Article: Purwa Rathi, Machine Borne Inventions \& Ownership: A Patenting Perspective. International Journal of Intellectual Property Rights, 10(2), 2019, pp. 1520. http://iaeme.com/Home/issue/IJIPR?Volume=10\&Issue=2 


\section{INTRODUCTION}

AI is not a single technology, but a combination of many. When any of AI techniques, for example machine learning, fuzzy logic, probabilistic reasoning, neural network, SVMs, is utilized for creating a possibly novel and non-obvious solution to a real world problem, a patentable invention is borne. For the purposes of this study, such work is termed as AI enabled inventions (AEIs). Here, the deployment of human faculties towards creative contribution acknowledges a 'natural person/individual' as an inventor. Accordingly, prevailing laws on patentability that attribute exclusive rights to true and first inventor remains logically applicable, and any deviation in this context is rather unfounded. On the contrary, as the role of AI systems become predominant in invention making process to the extent of excluding human interference in any substantial way, elementary concern of true inventor ship arises. Imagine a situation in which an original creator of AI system is long gone, and the machine over the years has gained tremendous capability of performing a task more efficiently by self-learning and without being explicitly programmed. Agreeably, a natural person is not an inventor here. The focus, thence, will be to scout for the true inventor and analyze in granular detail what happens later. Such works exclusively borne out of machine's doing will be referred as Machine borne inventions (MBIs) in this study. The scope of discussion shall be limited to nuanced analysis of inventorship and ownership concepts, patentable elements and patentability standards, and probable patent regulations that can be designed to cater to such AEIs and MBIs. Further, the attempt is to address pertinent questions raised by various Patent Offices (primarily US Patent \& Trademark Office) regarding evaluation of existing guidelines for AI patent applications.

\section{INVENTOR OF AI INVENTION}

In the present context, it is important to identify peculiar patentable elements for both AEIs \& MBIs and bifurcate incoming inventions in either of these branches. The reason being simply that subject matter involving human intervention demands a differential treatment from the ones that are plainly and solely machine borne, when viewed from a legal standpoint. It will be way off base if someone is rewarded for innovation one never indulged in.

Let's find a probable solution ahead. An initial assessment whether an invention falls into one of the categories- either AEIs or MBIs is necessitated at first. In cases where the human involvement has contributed towards an invention making process (in whole or in part) as defined by reference to the language of claims, inventorship can be accredited to the individual fulfilling the inventorship criteria as per the general patent rule. Consequently, an individual claims all benefits and entitlements accompanying an inventor under present patent framework. In contrast, where the act of invention is entirely a machine effort- an autodidact one, a new taxonomy defining the true creator would be more desirable. Since the MBIs are beyond initial conception and predictability of original inventor, titlehood directed towards machines that systematically and innovatively solves real world problems seems to be a more lawful measure. Speaking plainly, there should be an opportunity to confer legal personhood, perhaps inventorship, upon such electronic personality. Demarcating inventorship between individual and electronic personality will not only explicitly establish authenticity of true originator of relevant work, but also eliminate undue need to admit improper titles.

However, status quo of inventorship is settled based on disclosure made in a patent document. As a basic rule, true inventor has to disclose in full detail the particulars of his invention along with advantages and practical utility accompanying it. Where a human is considered as an inventor, the disclosure shall entail all probable and foreseeable capabilities and benefits derivable from its use. After all, this would be one of the primary reasons for acknowledging one as an inventor. On the other hand, all that remaining unlisted, 
unforeseeable and unpredictable may be envisaged as a part of machine endeavor. Under such circumstances, the electronic personality could be assumed to have all patent rights and obligations as a human.

\section{INTRODUCING CONCEPT OF OWNERSHIP FOR ELECTRONIC PERSONALITY}

Along being an inventor, the machine is assigned with other associated denominations, including that of ownership. The concept of ownership for MBIs have potential implications in realms of patent law. Agreeably, AI machines may not hold any intangible rights per se; they cannot be trusted with any accountability as well. Still, it will be too vague to undermine the significance of conferring distinct inventorship and ownership rights to natural and electronic personality, just for the reason that it is too far-reaching giving meaning to such concept. This problem, if not remedied today, will lead to disorderliness of tomorrow. Following paragraphs propose a workable course of action in designating ownership titles for such MBIs.

At the outset, the jurisprudence of current patent regime can be tweaked to establish causal link between the owner and inventor. From this perspective, ownership can be accorded to individuals undertaking the task of producing and presenting the application on behalf of machine before the patent office. This can, unequivocally, be an original inventor, assignee, manufacturer, seller or anyone who is interested in furthering patent rights granted upon the beneficiary in lieu of certain disclosure. Since patent scheme is a quid pro quo arrangement, along with the rights flow legal risks associated with claimed ownership. The person interested in deriving profits from ownership of such machine shall also be ready to bear penalties and liabilities underlying such legal risks. The assessment has to be thorough and ownership fully documented. Though the whole scheme may appear to follow existing trends, yet the concern regarding willingness of an inventor to own a patent laced with that kind of liabilities and uncertainties will continue to exist. For many, a Trade Secret may be a more viable option to muster profits with no strings attached. Some logical answers to these perplexing thoughts may be found in subsequent paragraphs.

\section{RE-INTRODUCING PRODUCT PATENT REGIME IN MBIS}

The above scenario may be divided into two sub scenarios i) where product and process patent is granted, and ii) where only product patents are granted. Before delving deeper into tradeoffs of above two scenarios, let us first understand which parts of AI are eligible for patent protection-

- Data selection and processing to be used as a raw material for machine; or

- Training process that machine undergoes to deliver appropriate results; or

- Mathematical Models or algorithms that are tied to these machines to make them suitable for specific real world application

- A special purpose AI machine capable of executing algorithms or models in a technical and non-routine manner to produce a practical effect.

Apart from these elements, the ingenuity in filing of AI related patents may reside in a-

- Computer scientist who designs and executes machine learning algorithms on machines

- A data scientist who innovatively selects, prunes and labels training data used to train a model for AI; a selection that does not merely constitute a mental act, but an autonomous system that can perform this task in a specific way

- An engineer who is capable of identifying and integrating the output of machine into real world application 
- Or a combination thereof

AI based inventions make use of models and networks that evolve as a part of training process and are probabilistic in nature. This processing of using complex mathematical models to train the system are safely enclosed within its hidden layers. Further, the fundamental building blocks of AI i.e. mathematical models are not eligible for patent protection; though once they have technical character or exhibit technical improvement, they are fit to be se. However, it is almost virtually impossible for an individual to discover what is routine information and processing for a typical self-taught machine. If the solution is integrated with real world application-expressly the outcome or technical effect of such technical solution, it is likely to qualify as a patentable subject matter. But instead, the end result that proposes a solution to particular problem of real world can be discerned regardless of the training process it undergoes, which more or less remains imperceptible, the solution still stands a chance of seeking patent protection.

Therefore, by reinstituting the faith in product patent regime for MBIs, the owners can be stimulated to file for product patents and claim benefits. Infact, product patent will offer a higher level of protection as there not be any other competitor for same product. In parallel, relentless patent filing to match exceedingly high rate of innovation in MBIs can also be overtly monitored and checked. Considering the accelerated growth in MBIs, a possible scenario may exists, where small number of owners patent too many inventions too quickly, almost monopolizing the entire set up. To evade this speculated imbalance, it will be favorable to restrict the term of grant for product patent in MBIs, say for example, 7 years. This reasonable period of protection should neither seem burdensome for owners carrying weight of legal uncertainties, nor permit selected few to control markets for a prolonged 20year term, especially considering the pace with which this domain is progressing.

\section{PATENTABILITY STANDARDS FOR MBIS}

As the concept of "only product patents" for MBIs gains further pace, it is exposed to another intriguing level of complexity- deciding patentability standards for MBIs in addition to adjudicating machine's capability when assessed by human efforts. If the answer is not a strict no, it is also not an absolute yes. But patentability demands the otherwise. It has to be an absolute yes for a full proof examination. Candidly, it will be beyond the mental faculties of humans, in general, to fairly and squarely scrutinize capabilities of MBIs performing extraordinarily complex computations within its series of network layers. With the complexities of mathematical models unfolding in those hidden layers, the novelty and obviousness of its unique output can be fairly assessed only by an equivalently capable electronic personality having skill in art, suitably coined as 'EPHOSITA'.

As PHOSITA (person having ordinary skill in art) is for AEIs, EPHOSITA (electronic personality having ordinary skill in art) may be for MBIs. The enormous volumes of AI inventions gaining popularity over these years and the sprint with which they are aced will, sooner rather than later, necessitate the need for developing a machine probing machine. Inducting EPHOSITA will also reduce the burden of investigating magnitude of patent applications that will be rampantly filed by spirited innovators in swelling domain of AI. Additionally, PHOSITA and EPHOSITA are not likely to have their roles interchangeably played either. Neither EPHOSITA be inspecting applications filed by individuals for the grounds of obviousness will be too occasional and germane; nor PHOSITA be roped in for determining capabilities of MBIs, the premise being considerably different in each case.

However, with no EPHOSITA existing today, way forward is to bring one. Introducing PHOSITA will be posed with several severe challenges- making EPHOSITA functional; deciding standards of equivalence; most importantly examining the status of patent 
applications filed in the interval between filing date and date of inducting EPHOSITA (a transitional phase). The road ahead towards building a functional and equivalently capable PHOSITA does not seem to be bothersome for much talented pool of experts including software developers, thinkers, technologists or lawmakers. Collaboratively, they can develop and deliver an electronic personality that can skillfully master the art of assessing novelty and obviousness features of MBIs. A discreet set of rules and procedures shall be, however, required for examination of applications filed in between the transitional phase.

Well, one approach can be to reinstate filing of mailbox application. The solution is simple and well known to all. Simply, it implies that until EPHOSITA becomes operational, MBIs related applications can be filed as a mailbox application to gain exclusive marketing rights (EMRs) for a determined period of time. Such applications can be kept pending in the mailbox or blackbox which can be opened after a predetermined time, say for example until 2021-whenever EPHOSITA is fit to be launched. During this transitional phase, EMRs can be provided to all those applicants whose applications are lying in mailbox. On and from the date of the publication of the application for patent and until the date of grant of patent in respect of such patents, the applicant may have like privileges and rights as if the patent has been granted on date of publication of application. This deferred examination and provisional protection will be equally advantageous for both the parties. While the applicants can secure a priority date for themselves, the examination can be appropriately performed as and when an equivalent EPHOSITA is operative within the Patent Office.

As has been demonstrated throughout this study, the current legal and regulatory framework may not be suitable for addressing inventorship, ownership and patentable rights of MBIs simply because MBIs are purely machine created, beyond human comprehension, and require a distinct entity to equitably decide for its patentability. Hence, while the present legal regime be reserved for AEIs or human facilitated inventions, a new framework may be devised for MBIs with inclusions of EPHOSITA and provisional EMRs for a fixed tenure.

\section{CONCLUDING REMARKS}

The foregoing discussion establishes the growing need for a new mechanism of making MBIs meaningfully patentable with inventorship and ownership rights respectively attributable to its original creator and to one capable of bearing ownership risks. Also that the newly crafted creations cannot be unfittingly casted in molds of existing patent order. Although the proposed solution of introducing EPHOSITA, reinstituting product patent regime, provisioning EMRs may appear burdensome and too demanding for now, but the quicker we respond to these technological advancements, safer we would be treading in these unchartered waters. The product patent regime will prevent flooding of patent rights for MBIs with unfenced boundaries as the process of realizing the final outcome will always remain a subject of contention. Meanwhile during the arduous course of creating EPHOSITA, transient provisions of EMRs will secure priority dates for applicants besides granting the owners the right to sell and distribute their inventions until substantive examination. To conclude, new directives are necessitated for comprehensively addressing numerous patent law issues for MBIs involving AI activity both at present and in foreseeable futures of technological development. 


\section{REFERENCES}

[1] Dr. Noam Shemtov, A study of inventorship in inventions involving AI activity, https://www.epo.org/news-issues/issues/ict/artificial-intelligence.html\#study

[2] Tracey L. Thompson, USPTO, Federal Register, August 27, 2019, https://www.govinfo.gov/content/pkg/FR-2019-08-27/pdf/2019-18443.pdf

[3] World Economic Forum, Artificial Intelligence Collides With Patent Law, April 2018, http://www3.weforum.org/docs/WEF_48540_WP_End_of_Innovation_Protecting_Patent _Law.pdf

[4] Timothy Chou and Philip H. Albert, When AI Creates AI -- Who Owns the Rights?, September 9, 2019, https://www.dwt.com/blogs/artificial-intelligence-lawadvisor/2019/09/uspto-comments-on-ai-patent-applications \#page $=1$ 